\title{
Derivation of consolidated normal reference values for right and left ventricular quantification by cardiac magnetic resonance using a novel meta-analytic approach
}

\author{
Yang Zhan ${ }^{1}$, Jaime L Shaw ${ }^{2}$, Michael Chetrit ${ }^{1}$, Michele Arcopinto ${ }^{2}$, Russell Steele ${ }^{1}$, Michael L Chuang ${ }^{2}$, \\ Warren J Manning ${ }^{2}$, Jonathan Afilalo ${ }^{1 *}$
}

From 19th Annual SCMR Scientific Sessions

Los Angeles, CA, USA. 27-30 January 2016

\section{Background}

Although cardiac magnetic resonance (CMR) has superior accuracy and reproducibility for quantification of ventricular size and function, its interpretation is limited by non-robust normative data derived mainly from small single-center studies.

\begin{abstract}
Methods
We performed a systematic review of the literature from 2003-2015 to identify all published studies that measured right and left ventricular parameters by CMR in healthy adult controls. The parameters of interest were: end-diastolic volume, end-systolic volume, stroke volume, ejection fraction, and mass (indexed to body surface area). Inclusion criteria were: SSFP-based acquisition and quantification on a short-axis stack using the method of discs. Exclusion criteria were: enrollment of non-healthy subjects, trained athletes, or overlapping data with other publications. Queries related to overlapping data were resolved by contacting the authors of the studies. We extracted age- and sex-specific data when available and stratified according to whether the papillary muscles were traced or not. We also accounted for the distribution of each parameter (rather than assuming a normal distribution), which was determined from a subset of individual patient-level data. Two observers reviewed the studies in duplicate and a third observer audited discordances. We performed a random-effects meta-analysis of the data (using the same approach
\end{abstract}

'Jewish General Hospital, McGill University, Montreal, QC, Canada Full list of author information is available at the end of the article developed for the American Society of Echocardiography chamber quantification guidelines) to generate pooled mean values for each parameter, upper reference values, lower reference values, and 95\% confidence intervals surrounding each of these values.

\section{Results}

Our PubMed search strategy identified 900 potentially relevant studies, of which 305 were eligible based on the pre-specified inclusion and exclusion criteria. The number of included studies ranged from $220(\mathrm{~N}=8,416$ healthy subjects) for left ventricular ejection fraction to 28 ( $\mathrm{N}=993$ healthy subjects) for right ventricular mass index. There were consistently fewer studies for right- as compared to left-sided parameters. There was a balanced proportion of males and females, and age ranged from 18 to 80 years. The normal reference values generated by our meta-analysis models are presented in Table 1 along with their 95\% confidence intervals. When models were stratified by papillary muscle tracing, the only notable difference observed was for right and left ventricular mass index; as expected, mass was significantly greater when papillary muscles were included as part of the measured myocardial mass. Further analysis of age- and sex-specific differences in normative data was performed by metaregression and will be presented.

\section{Conclusions}

The consolidated reference values generated by our meta-analytic approach have provided robust estimates of upper and lower limits of normal that reflect the 
Table 1 Normal Reference Values for Right and Left Ventricular Parameters by CMR

\begin{tabular}{|c|c|c|c|c|c|}
\hline Parameter & Studies & Subjects & Lower Limit & Mean Value & Upper Limit \\
\hline LVEDVI & 123 & 5730 & $53(51,56)$ & $76(75,78)$ & $99(96,101)$ \\
\hline LVESVI & 96 & 4831 & $15(14,16)$ & $28(27,28)$ & $40(39,41)$ \\
\hline LVSVI & 70 & 4020 & $35(33,37)$ & $49(48,50)$ & $63(61,65)$ \\
\hline LVEF & 220 & 8416 & $53(53,54)$ & $64(63,64)$ & $74(73,75)$ \\
\hline LVMI & 127 & 6182 & $39(36,41)$ & $57(55,59)$ & $75(72,77)$ \\
\hline RVEDVI & 65 & 2077 & $57(53,61)$ & $82(80,85)$ & $108(104,111)$ \\
\hline RVESVI & 56 & 1688 & $20(17,22)$ & $35(33,36)$ & $49(47,52)$ \\
\hline RVSVI & 39 & 1201 & $33(30,36)$ & $47(46,49)$ & $62(59,64)$ \\
\hline RVEF & 83 & 2360 & $47(45,48)$ & $58(57,59)$ & $69(67,70)$ \\
\hline RVMI & 28 & 993 & $13(11,16)$ & $20(18,22)$ & $27(24,29)$ \\
\hline
\end{tabular}

* If papillary muscle were vs. were not traced, upper limit was 79 vs. 66 for LVMI and 35 vs. 19 for RVMI, respectively. Parentheses denote $95 \%$ confidence intervals surrounding point estimates. Abbreviations: LV, left ventricle; RV, right ventricle; EDVI, end diastolic volume index (mL/m2); ESVI, end systolic volume index $(\mathrm{mL} / \mathrm{m} 2)$; SVI, stroke volume index $(\mathrm{mL} / \mathrm{m} 2)$; $\mathrm{EF}$, ejection fraction (\%); MI, mass index ( $\mathrm{g} / \mathrm{m} 2)$.

accumulated body of published data for right and left ventricular quantification using SSFP-based CMR.

\section{Authors' details}

${ }^{1}$ Jewish General Hospital, McGill University, Montreal, QC, Canada. ${ }^{2}$ Beth

Israel Deaconess Medical Center, Harvard University, Boston, MA, USA

Published: 27 January 2016

- Convenient online submission

- Thorough peer review

- No space constraints or color figure charges

- Immediate publication on acceptance

- Inclusion in PubMed, CAS, Scopus and Google Scholar

- Research which is freely available for redistribution 